REVESCO. Revista de Estudios Cooperativos

ISSN: $1885-8031$

http://dx.doi.org/10.5209/REVE.59774

\title{
Análisis comparativo de centrales de compras cooperativas: problemas y soluciones
}

\author{
Carmen Boccatonda ${ }^{1}$, Lucía Clara Banchieri ${ }^{2}$ y Fernando Campa Planas ${ }^{3}$
}

Recibido: 6 de junio de 2017 / Aceptado: 14 de marzo de 2018

Resumen. El objetivo del estudio consiste en identificar los problemas a los que se han enfrentado las centrales de compras cooperativas en las diferentes etapas de su ciclo de vida. La metodología utilizada es el estudio de casos. El muestreo son dos casos específicos que son escogidos por ser reveladores y ejemplos extremos. Por un lado, la CCFACC, una incipiente central de compras cooperativa argentina, y, por el otro, la Coop Italia, un modelo con amplia trayectoria en el mercado, con resultados comprobados y con proyecciones de mayor crecimiento. Además, resulta interesante que cada uno de los casos se ubica contextos y continentes diferentes. La identificación de los problemas de las centrales de compras cooperativas diferenciados por etapa del ciclo de vida, así como las formas de solucionarlos, en caso de encontrarlas, aportan a mejorar el desempeño del movimiento cooperativo. Se identifican claramente los problemas que sufren las centrales de compras cooperativas con el fin de poder conocerlos y tomar acciones para evitarlos en el caso de una nueva experiencia. El presente trabajo ha aportado a la bibliografía el estudio de dos casos de centrales de compras cooperativas situadas en contextos diferentes y en distintas fases del ciclo de vida, distinguiendo los problemas enfrentados y las formas de solucionarlos.

Palabras clave: Cooperativas de consumo; Central de compras; Estudio de casos.

Claves Econlit: L31.

\section{[en] Comparative analysis of cooperative purchasing centers: problems and solutions}

\begin{abstract}
The aim of the study is to identify the problems faced by cooperative purchasing centers during the different stages of their life cycle. Design/methodology: The methodology used is the case study. The cases chosen correspond to two specific cases revealing extreme examples. On one hand the CCFACC, an incipient Argentine cooperative purchasing center, and, on the other, Coop Italia, a model with a long history in the market, with proven results and projections of great growth. In addition, it is interesting that each of the cases is located on different contexts and continents. The identification of the problems of the cooperative purchasing centers differentiated by stage of the life cycle, as well as the ways of solving them, if found, can contribute to improve the performance of the cooperative movement. The problems experienced by cooperative purchasing centers are clearly identified in order to be able to meet them and take action to avoid in the case of a new experience. The present research has contributed to the bibliography about cooperative purchasing centers located
\end{abstract}

1 Universidad Nacional del Sur, Argentina

Dirección de correo electrónico: carmenboccatonda@gmail.com.

2 Universidad Nacional del Sur, Argentina

Dirección de correo electrónico: lucia.banchieri@uns.edu.ar.

3 Universitat Rovira i Virgili, España

Dirección de correo electrónico: fernando.campa@urv.cat. 
in different contexts and in different phases of the life cycle, distinguishing the problems faced and the ways to solve them.

Keywords: Consumer cooperatives; Central purchasing; Case studies.

Sumario. 1. Introducción. 2. Marco teórico. 3. Metodología. 4. Resultados. 5. Conclusiones. 6. Referencias bibliográficas.

Cómo citar: Boccatonda, C., Banchieri, L.C. y Campa Planas, F. (2018) Análisis comparativo de centrales de compras cooperativas: problemas y soluciones. REVESCO. Revista de Estudios Cooperativos, Primer Cuatrimestre, No 127, pp. 26-44. DOI: 10.5209/REVE.59774.

\section{Introducción}

Resulta inevitable tener en cuenta la preocupación sobre la creación de estrategias de desarrollo sostenible en un contexto en donde la responsabilidad social empresaria es tema cotidiano. "Los fundamentos morales del capitalismo deben ser reconsiderados", sostiene Zsolnai (2010), al mismo tiempo que asegura que la competitividad es la ideología prevaleciente en el mundo económico actual. Es desde esta incapacidad del mercado y del sector público de satisfacer determinadas necesidades sociales en donde tienen lugar las organizaciones de la Economía Social, donde las cooperativas adquieren mayor significación histórica.

Justamente, uno de los tres objetivos de la Alianza Internacional de Cooperativas (en adelante, ACI) en su Plan para una Década Cooperativa (2013) es ser líder reconocido en cuanto a la sostenibilidad económica, social y medioambiental, refiriéndose al movimiento cooperativo. El sexto principio cooperativo conlleva la expresión práctica del valor cooperativo de solidaridad y es el compromiso con la cooperación cooperativa el hecho distintivo de este tipo de organizaciones.

"En los modernos mercados globalizados y en las complejas cadenas de suministro actuales, resulta crucial que las cooperativas de todos los sectores trabajen juntas para maximizar la ventaja cooperativa", aclara la ACI (2013) sobre el sexto principio cooperativo, dando lugar a la formación de una central de compras cooperativa.

Una forma de integración para las cooperativas de consumo son las centrales de compras, entendiendo por central de compras un operador que, disponiendo de recursos económicos y personalidad jurídica propia, tienen por objetivo desarrollar las actividades y prestar servicios a las empresas independientes que, con espíritu de cooperación, se han asociado a la organización mediante reglamentación interna con el objetivo de mejorar su competitividad, según la Asociación Nacional de Centrales de Compras y Servicios de España (ANCECO, 2017).

Pereira et. al. (2010) afirman que hacen faltan estudios que colaboren en el establecimiento de los problemas que afectan a las redes de compras para que las mismas puedan perdurar en el tiempo. Específicamente, sostienen que hacen falta investigaciones en diferentes contextos y sectores.

Este estudio tiene como principal objetivo identificar los problemas a los que se han enfrentado dos centrales de compras cooperativas, la Coop Italia y la Central de Compras de la Federación Argentina de Cooperativas de Consumo (en adelante, 
CCFACC). Las centrales de compras elegidas están ubicadas en países con realidades completamente diferentes y pertenecen a un sector distinto, el cooperativismo, que el estudio de Pereira et al (2010).

El presente trabajo se estructura en cinco secciones. En primer lugar, se identifican en la literatura los problemas a los que se enfrentan las centrales de compras. A continuación, se desarrolla la metodología utilizada, el estudio de casos, y se describen las unidades de análisis. En tercer lugar, se realiza un análisis comparativo de los problemas hallados en ambas centrales de compras escogidas, teniendo en cuenta que la CCFACC es un proyecto incipiente con apenas meses de funcionamiento y la Coop Italia tiene amplia trayectoria y resultados satisfactorios, desarrollándose cada una en contextos geográficos y socioculturales distintos. Por último, se exponen las conclusiones del trabajo realizado.

\section{Marco teórico}

\subsection{Integración y centrales de compras}

Sobre la integración de compras, Acosta y Verbeke (2009) asienten que "la cooperación entre empresas es un recurso estratégico en el mundo empresarial para responder eficazmente a un entorno competitivo" (p. 2). Adicionalmente, Charterina (2011), en su análisis de los principios cooperativos, concluye que el sexto principio

Las asociaciones son más adaptables que las estructuras de las grandes corporaciones y poseen la capacidad de reacomodarse con mayor facilidad a las necesidades y exigencias del mercado. Adicionalmente, se reconoce que la asociación no es una oportunidad más entre otras posibles para las cooperativas, sino que es una manera natural de operar debido a su naturaleza solidaria (Menzani y Zamagni, 2010).

Para Heinz et. al. (2014) los beneficios de una central de compras son:

- Mayor poder de negociación

- Combinación de know how entre empresas

- Almacenamiento conjunto

- Marketing homogéneo

- Reducción de precios

- $\quad$ Prorrateo de costes

- $\quad$ Reconocimiento de marca

- Mejora en la logística

- Modernización de la estrategia

- Competitividad

- $\quad$ Mejora del servicio al cliente

- Especialización de los compradores

- Acceso a proveedores

- $\quad$ Reducción del número de operaciones

Acentuando estos beneficios, Barberini (2009), en referencia a la experiencia de la Coop Italia, argumenta que el cooperativismo de consumo italiano ha mejorado los servicios a sus asociados. Por ejemplo, ha habido un progreso en los puntos de 
venta, en relación a que se ha ido encontrando el tamaño y la ubicación más apropiada, así como se ha mejorado el surtido ofrecido, ya que muchas veces éste resultaba limitado o no se ajustaba a las necesidades de los consumidores.

\subsection{Ciclos de vida}

Con el fin de comprender el proyecto de una central de compras como un proceso madurativo, Pousa (2006) establece diferentes fases del ciclo de vida de las centrales de compra, los cuales se caracterizan por quién realiza la gestión, el área geográfica en la cual se desempeña, sus objetivos y alguna característica adicional.

Cuadro. 1. Ciclo de vida de centrales de compras

\begin{tabular}{|c|c|c|c|c|}
\hline Etapa & Gestión & $\begin{array}{c}\text { Área de } \\
\text { actuación }\end{array}$ & Foco & $\begin{array}{c}\text { Principal } \\
\text { característica }\end{array}$ \\
\hline Precursora & $\begin{array}{l}\text { Realizada por } \\
\text { los propios } \\
\text { asociados }\end{array}$ & $\begin{array}{l}\text { Micro } \\
\text { regiones }\end{array}$ & $\begin{array}{l}\text { - Conseguir } \\
\text { descuentos y } \\
\text { plazos de compra }\end{array}$ & $\begin{array}{l}\text { - Intercambio } \\
\text { de } \\
\text { información } \\
\text { entre los } \\
\text { asociados }\end{array}$ \\
\hline Desarrollo & $\begin{array}{l}\text { Realizada por } \\
\text { los propios } \\
\text { asociados, con } \\
\text { más tiempo de } \\
\text { dedicación }\end{array}$ & Regional & $\begin{array}{l}\text { - Compra } \\
\text { conjunta } \\
\text { - Mayor apoyo y } \\
\text { beneficio de } \\
\text { proveedores }\end{array}$ & \begin{tabular}{l}
\multicolumn{2}{c}{ Razonable } \\
grado de \\
integración de \\
información \\
entre los \\
asociados \\
- \\
Incorporación \\
de servicios de \\
marketing y \\
finanzas
\end{tabular} \\
\hline Maduración & $\begin{array}{lr}\text { Dedicación } & \\
\text { exclusiva } & \text { de } \\
\text { empresario } & \mathrm{o} \\
\text { personal } & \\
\text { contratado } & \end{array}$ & $\begin{array}{l}\text { Varias } \\
\text { regiones }\end{array}$ & $\begin{array}{lr}\text { - Ampliación de } \\
\text { los recursos } \\
\text { ofrecidos r de } \\
\text { marketing, } \\
\text { finanzas r y } \\
\text { recursos humanos }\end{array}$ & $\begin{array}{l}- \\
\text { Formalización } \\
\text { de procesos } \\
\text { - Mejora de la } \\
\text { logística, } \\
\text { posible } \\
\text { abastecimiento } \\
\text { centralizado }\end{array}$ \\
\hline
\end{tabular}




\begin{tabular}{|c|c|c|c|c|}
\hline $\begin{array}{l}\text { Madurez } \\
\text { plena }\end{array}$ & $\begin{array}{l}\text { Gestión } \\
\text { altamente } \\
\text { profesional, } \\
\text { con sólidos } \\
\text { conocimientos } \\
\text { en } \\
\text { planeamiento } \\
\text { estratégico }\end{array}$ & $\begin{array}{ll}\text { Nacional } & \mathrm{o} \\
\text { varias } & \\
\text { regiones } & \end{array}$ & $\begin{array}{l}\text { - Integración } \\
\text { tecnológica con } \\
\text { proveedores } \\
\text { - Sistemas de } \\
\text { información } \\
\text { - Logística } \\
\text { integrada } \\
\text { - Pago } \\
\text { centralizados }\end{array}$ & $\begin{array}{lr}\text { - Se } & \text { puede } \\
\text { llegar a la } \\
\text { creación } & \text { de } \\
\text { empresas, en } & \text { donde los } \\
\text { socios tienen } \\
\text { participación } \\
\text { accionaria }\end{array}$ \\
\hline
\end{tabular}

Fuente: Pousa (2006) en base a Souza (2004)

\subsection{Problemas hallados por diferentes experiencias de centrales de compras}

Por su parte, el estudio de Pereira et. al. (2010) tiene como objetivo dilucidar las razones por las cuales las empresas abandonan las centrales de compras, con el fin de contribuir a un mejor funcionamiento de las centrales para que puedan llegar a los objetivos estipulados. Mediante un estudio bibliográfico de producción científica sobre cierre de redes de empresas, concluyeron que las posibles causas de fracaso de las redes interorganizacionales son:

- $\quad$ Rivalidad entre las firmas.

- $\quad$ Riesgo de oportunismo.

- Retornos asimétricos.

- Desconfianza / poco compromiso.

- Complejidad gerencial.

- Dificultad de coordinación.

- $\quad$ Costes de agencia y burocracia. Rigidez organizacional.

- $\quad$ Dificultad en la gestión de cooperación.

Adicionalmente, Heinz et. al. (2014) también analizan los inconvenientes de incurrir en este tipo de asociación a través de un estudio de caso realizado sobre una red de compras brasileña con 35 supermercados regionales asociados. La central de compras estudiada fue escogida por haber sido considerada como caso de éxito por el Servicio Brasilero de Apoyo a Micro y Pequeñas Empresas. Los inconvenientes detectados fueron los siguientes:

- Constitución societaria.

- Lugar a comportamiento oportunista de los participantes.

- $\quad$ Dificultad en establecer mecanismos de control e incentivos.

- $\quad$ Lentitud en el proceso decisorio.

- Doble tributación.

- Asimetría de información.

- $\quad$ Diferencia de tamaño de los participantes.

- $\quad$ Proceso de gestión.

- Coste-beneficio.

- Conflicto de intereses.

- Cambio cultural. 
Por su parte, el trabajo de Xavier Filho et. al. (2013) también busca detectar las causas por las que la cantidad de redes de compras brasileñas disminuyó de 841 en 2008 a 778 en 2011. Mediante un trabajo exploratorio de material empírico, llegan a que los problemas más recurrentes son:

- Falta de asociativismo y cooperación (conflicto de intereses). Individualismo.

- $\quad$ Falta de compromiso por parte de los participantes.

- $\quad$ Análisis coste-beneficio.

- Diferencias culturales, regionales.

- Expectativa de resultados inmediatos.

- Falta de concientización de la importancia de la cooperación, falta de confianza.

- $\quad$ Problemas de gestión, falta de liderazgo de la red.

- Diferencia de tamaño de participantes.

- Oportunismo.

- Falta de apoyo del programa público.

En relación a este tema, en el taller de consumo de la IV Cumbre de las Américas, una central de compras cooperativa brasileña relató su experiencia y expuso los factores desestimulantes que les está impidiendo el buen funcionamiento de la central (Sena da Silva, 2016):

- Expectativas de precios bajos.

- Ausencia de cultura.

- $\quad$ Fuerza de marcas regionales.

- Costes de transportes.

- Impuestos.

- Volumen. Dependencia del número de locales.

- $\quad$ Surtido, falta de espacio.

En última instancia, durante el año 2016, la ANCECO realizó un estudio cualicuantitativo a 187 centrales de compras nacionales. Entre los resultados más importantes, es posible distinguir los siguientes aspectos:

- La principal amenaza revelada para las centrales de compras son sus propios socios. En este sentido, se señalan el egoísmo, la falta de compromiso e implicación de algunos socios que no siguen la consigna de la central, con la consiguiente pérdida de capacidad de negociación, facilitando la negociación directa con el proveedor evitando la relación con la central.

- Dentro de las líneas que marcarán la actividad de los próximos años, la amplitud de la propuesta de servicios, su calidad y la capacidad de adaptación de los servicios a las necesidades de los asociados, se destacan como las opiniones más compartidas.

- $\quad$ No todos los socios comparten los mismos intereses. En general, un grupo sí coincide, mientras que otro sólo se comporta de manera individualista y utiliza la central meramente por la negociación de acuerdos con proveedores. 
- La mayoría de los encuestados está de acuerdo en que el tamaño resulta un problema tanto por la heterogeneidad de los asociados, como por la concentración de los proveedores.

- $\quad$ El 50\% de los encuestados coincide en que el grado de implicación y fidelidad de los socios es bajo, mientras que el 40\% opina que es medio; y el resto, $10 \%$, alto.

- La mayoría de las centrales no dispone de un régimen sancionador o, en caso de tenerlo, nunca se aplica.

En síntesis, el Cuadro 2 contiene los problemas que deben afrontar las centrales de compras hallados por los diferentes autores. A continuación del mismo se expone una breve descripción de cada uno de ellos.

Cuadro. 2. Problemas de centrales de compras hallados por distintos autores

\begin{tabular}{|l|l|}
\hline Problema & Autores \\
\hline $\begin{array}{l}\text { Constitución societaria } \\
\begin{array}{l}\text { Dificultad de establecer } \\
\text { mecanismos de control e } \\
\text { incentivos }\end{array}\end{array}$ & $\begin{array}{l}\text { Heinz et. al. (2014), Pereira et. al. (2010) } \\
\text { ANCECO (2017) }\end{array}$ \\
\hline Doble tributación & Heinz et. al. (2014), Sena da Silva (2016) \\
\hline Asimetría de información & Heinz et. al. (2014) \\
\hline $\begin{array}{l}\text { Diferencia de tamaño de los } \\
\text { participantes / Volumen de } \\
\text { pedidos }\end{array}$ & $\begin{array}{l}\text { Heinz et. al. (2014), Pereira et. al. (2010), } \\
\text { ANCECO (2017), Xavier Filho et. al. (2013), } \\
\text { Sena da Silva (2016) }\end{array}$ \\
\hline $\begin{array}{l}\text { Proceso de gestión / Lentitud } \\
\text { en procesos decisorios }\end{array}$ & $\begin{array}{l}\text { Heinz et. al. (2014), Pereira et. al. (2010), } \\
\text { Xavier Filho et. al. (2013) }\end{array}$ \\
\hline Coste-beneficio & $\begin{array}{l}\text { Heinz et. al. (2014), Pereira et. al. (2010), } \\
\text { Xavier Filho et. al. (2013) }\end{array}$ \\
\hline Conflicto de intereses & $\begin{array}{l}\text { Heinz et. al. (2014), Pereira et. al. (2010), } \\
\text { ANCECO (2017), Xavier Filho et. al. (2013) }\end{array}$ \\
\hline Cambio cultural & $\begin{array}{l}\text { Heinz et. al. (2014), Pereira et. al. (2010), Sena } \\
\text { da Silva (2016), Xavier Filho et. al. (2013) }\end{array}$ \\
\hline $\begin{array}{l}\text { Falta de compromiso } \\
\text { Oportunismo / Rivalidad entre } \\
\text { las firmas }\end{array}$ & $\begin{array}{l}\text { Pereira et. al. (2010), ANCECO (2017), Xavier } \\
\text { Filho et. al. (2013), Heinz et. al. (2014) }\end{array}$ \\
\hline Expectativas de precios bajos & Sena da Silva (2016), Xavier Filho et. al. (2013) \\
\hline $\begin{array}{l}\text { Surtido / Fuerza de marcas } \\
\text { regionales }\end{array}$ & Sena da Silva (2016) \\
\hline Logística & Sena da Silva (2016) \\
\hline $\begin{array}{l}\text { Falta de apoyo del programa } \\
\text { público }\end{array}$ & Xavier Filho et. al. (2013) \\
\hline
\end{tabular}

Fuente: elaboración propia a partir de la revisión bibliográfica efectuada 


\section{Constitución societaria}

Inconvenientes legales y procesos burocráticos pueden complicar la constitución de la asociación, así como la afiliación de las empresas a la central. "La consolidación institucional es una condición para el funcionamiento, dado que genera estabilidad de las relaciones cooperativas para el desarrollo de las identidades comunes y el fortalecimiento de los vínculos" (Pereira et. al., 2010, p. 67).

Dificultad de establecer mecanismos de control e incentivos

Según el informe de la ANCECO (2016), la principal amenaza para las centrales son sus propios asociados. Por ende, es necesario un mecanismo de control, pero reconocen que no ejercen penalidades por temor a que abandonen la red.

\section{Doble tributación}

Al introducir un intermediario (rol de la central), por ejemplo, se puede dar lugar para la doble tributación: del proveedor a la central y de la central al asociado.

\section{Asimetría de información}

La condición de igualdad se debe dar en todo sentido. La transparencia es una de las claves para el buen funcionamiento del sistema. Según Adobor y McMullen (2014), el intermediario debe ser confiable y demostrar legitimidad, no distorsionar la información.

\section{Diferencia de tamaño de los participantes / Volumen de pedidos}

Aunque todas las cooperativas asociadas se encuentran en iguales condiciones para poder acceder a los productos ofrecidos, puede ser que el volumen que necesitan las más pequeñas no alcance el mínimo requerido y no pueda realizar el pedido. En el informe de la ANCECO (2016) se reconoce que la diferencia de tamaño es un problema por la heterogeneidad de los asociados, hecho también comprobado en la central brasileña (Sena da Silva, 2016).

\section{Proceso de gestión / Lentitud en procesos decisorios}

Las organizaciones grandes pueden tienen procesos lentos en el momento de tomar decisiones, ya que las principales decisiones de la red están subordinadas a la supervisión de todos los asociados (Heinz et. al., 2010). A su vez, los asociados pueden no estar conformes con la dinámica de la central para la gestión del proceso.

\section{Coste-beneficio}

Siguiendo a Pereira et. al. (2010), una de las claves para que las empresas sigan apostando por la central es que las ganancias provenientes de la cooperación sean superiores, a largo plazo, a los beneficios que pueden ser obtenidos a corto plazo. 


\section{Conflicto de intereses}

Tal como se describe en Heinz et. al. (2014), la participación en una central de compras exige que los asociados tengan espíritu colectivo, dado que se trabaja en la búsqueda del bien común. Puede suceder que haya falta de conciencia de los beneficios a obtener a largo plazo y que cada uno actúe de forma individualista, atentando contra el espíritu cooperativo. Este problema se puede interpretar también como una consecuencia del análisis coste-beneficio.

\section{Cambio cultural}

El estudio de Xavier Filho et. al. (2013) concluye que el choque cultural es un factor determinante para el abandono de una asociación. La resistencia a los cambios de hábitos es un elemento que se verifica ex-post y que tiene el potencial de apoyar o desistirse ante la cooperación.

\section{Falta de compromiso / Oportunismo / Rivalidad entre las firmas}

Según Pereira et. al. (2010), "los beneficios de la cooperación son inciertos y orientados para el futuro, al tiempo que las acciones oportunistas ofrecen posibilidades de ganancia presente" (p. 72).

Por otro lado, resaltar el planteamiento de Xavier Filho et. al. (2013), en cuanto a que algo no perceptible por la economía tradicional está posibilitando arreglos organizacionales competitivos, basados en lazos subjetivos que envuelven valores socialmente construidos, como la confianza y el respeto.

\section{Expectativa de precios bajos}

Muchas veces, un menor precio se consigue con volumen, punto conflictivo en una etapa inicial del proyecto. Sobre este tema, se recurre nuevamente a las expectativas a corto plazo que no llegan a distinguir la necesidad de madurez de un proyecto para poder lograr los objetivos deseados.

\section{Surtido / Fuerza de marcas regionales}

Es posible que cadenas regionales estén afianzadas a su área de influencia y comercialicen con productores de la zona, hecho que entra en juego cuando el negocio se abre y se comparte el poder de decisión. Este hecho puede provocar desencanto por parte de los asociados.

Por otra parte, el surtido ofrecido por la central puede no cumplir con las expectativas y necesidades de los asociados y significar un problema para la comercialización e influir directamente en la competitividad.

\section{Logística}

Según Sena da Silva (2016), la logística ha sido un factor clave para el fracaso de la central brasileña, influido tanto por factores internos, como la organización de los almacenes, por ejemplo, como por factores exógenos como pueden ser las distancias y el estado de las vías de transporte. 


\section{Falta de apoyo del programa público}

El apoyo gubernamental se vuelve fundamental al momento de encarar este tipo de proyectos. Tanto el marco jurídico, como las normas tributarias y comerciales terminan delimitando el campo de acción de las asociaciones.

\section{Metodología}

Según Hernández Sampieri (2010), "los estudios exploratorios se efectúan, normalmente, cuando el objetivo es examinar un tema o problema de investigación poco estudiado o que no ha sido abordado antes". En particular, no se ha encontrado literatura previa sobre los problemas de las centrales de compras cooperativas.

El uso del estudio de caso ofrece importantes resultados e información que no es posible encontrar por medio de los métodos cuantitativos y que es muy valiosa para la toma de decisiones en empresas. Además, Yacuzzi (2005) sostiene que este tipo de metodología se enriquece más aún cuando se fomenta el diálogo entre la epistemología y las acciones de campo, al mismo tiempo que Gummesson (1991) asegura que el estudio de caso es cada vez más aceptado como una herramienta científica en la administración de empresas.

Un aspecto no menor es el diseño del estudio de caso. Siguiendo a Yin (2001), este trabajo se podría clasificar como un estudio de casos múltiples integrados, en el sentido que hay más de una unidad de análisis (dos en este caso) que se estudian relacionalmente en referencia al interrogante inicial que da inicio a la investigación.

A continuación, se detallarán las unidades de análisis, las fuentes de información y el análisis de datos, componentes fundamentales del estudio de caso según Yin (2001).

\subsection{Unidades de análisis}

Las centrales de compras elegidas se encuentran en diferentes etapas del ciclo de vida. La Coop Italia está en una fase madura lo que permite relevar mayor número de experiencias por el tiempo que hace que funciona. Por su parte, investigar los problemas a los que se enfrenta la CCFACC resulta de vital importancia, ya que es probable que cualquier central de compras los verifique en sus inicios, siendo indispensable su solución para que la central perdure en el tiempo.

\section{Coop Italia}

La Asociación Nacional de Cooperativas de Consumo italianas (en adelante, ANCC) fue constituida en 1955, constituyendo la representación nacional del movimiento cooperativo. "Coop Italia se constituyó el 1 de enero de 1968 y asumió la función de compras, marketing, proyección y gestión de la logística, la producción directa y otras actividades de asistencia a las cooperativas" (Barberini, 2009).

A su vez, en 1999 se unifica la central de compras con la central de marketing, denominada Coop Italia. Con la creación de la central, se pretendía ofrecer los 
medios para ejercer una estructura formalizada de comercialización, con la intención de evitar la concentración de poder en el mercado.

En cuanto a la distribución de responsabilidades, de acuerdo a Migliavacca (2016), se distinguen las siguientes entidades:

- ANCC: organismo de representación político-institucional de las cooperativas de consumo italianas.

- COOP ITALIA: consorcio para las compras y políticas de marketing, para la definición de las estrategias de producción y marca propia.

- INRES: instituto nacional de asesoramiento, diseño e ingeniería que proyecta la estructura de ventas. Se encarga de realizar el diseño de los supermercados (layouts), la organización interna y prepara los modelos de contrato con proveedores, entre otras actividades.

- SCUOLA COOP: consorcio encargado de la actividad didáctica, de búsqueda y trato con el personal, con el objetivo de difundir la cultura del movimiento cooperativo.

De acuerdo a Migliavacca (2016), actualmente, el cooperativismo de consumo italiano consta de 93 cooperativas, que suman 1.165 puntos de venta y 1.670 .588 $\mathrm{m} 2$ de área de venta. Por otro lado, totaliza 8,5 millones de asociados, y 12.400 millones de euros de facturación anual.

La Coop se compone por 7 grandes cooperativas (que concentran el $95 \%$ de la facturación total de la cadena), 8 cooperativas medianas y 76 pequeñas cooperativas distribuidas a lo largo de todo el territorio nacional (16 regiones). A su vez, estas cooperativas se reúnen en consorcios, que son agrupaciones regionales conformadas por más de una cooperativa.

En cuanto a la clasificación de los puntos de venta, los Híper (más de 4.000/4.500 m2) se denominan Hipercoop; los Súper (entre 1.000 y $4.000 \mathrm{~m} 2$. de área de venta) se denominan Coop; los locales pequeños, con menos de $1.000 \mathrm{~m} 2$ de área de venta, se identifican como Hincoop. En este sentido, se puede identificar una estrategia de marketing homogéneo, por el cual se identifica con el mismo nombre a cualquier supermercado cooperativo italiano.

Por otro lado, salvo las campañas nacionales organizadas por la Coop; los precios de venta a los asociados son fijados libremente por cada cooperativa.

Resulta interesante detallar las estrategias elegidas por la organización, que condujeron al crecimiento de estos últimos años (Migliavacca, 2016):

Conveniencia: mejor oportunidad de ahorro, seguridad y ética del producto, frescura y compromiso cotidiano con los consumidores, apoyándose, sobre todo, en los productos de marca propia. Se pretende ofrecer productos de calidad con diferencias de precios importantes $(30 \%$ por debajo de las primeras marcas).

- Diferencia: defiende el precio justo, apoya la salud y lucha contra la mala alimentación, apuesta por la calidad de los alimentos y al desarrollo sostenible. El desarrollo de la marca propia es la clave de dicha estrategia.

Innovación: tanto de la comida, como del estilo de vida y consumo, la información, la conciencia del consumidor, la relación con el consumidor y de la manera de interpretar el mercado. 
Territorio: entendiendo a éste como un valor social, económico y cultural, persiguiendo aprovechar la potencialidad de la totalidad del territorio.

Según cifras de 2016, las cooperativas participan con el 19\% de las ventas del canal híper y supermercados y el $14,5 \%$ de las ventas totales del país (la mayor participación con respecto a otros grupos).

Las marcas propias de la Coop Italia son 7, entre las que se encuentran: Coop (2.700 referencias), Fiorfiore (400) y Vivi Verde (600). Las marcas propias representan el $26 \%$ de las ventas en sus locales y tienen como objetivo llegar al $30 \%$. Para la distribución de estos productos, disponen de 280 proveedores. Comparando la evolución de los productos marca propia con la del total del mercado, se observa una tendencia de crecimiento similar en los dos casos.

Por otro lado, se verifica una nueva asociación internacional de la Coop con Copernic, alianza de distribuidores independientes europeos, con sede en Bruselas. La misma involucra 4 redes de cooperativas europeas, las cuales abarcan 22 países, acumulan más de 22.000 puntos de ventas y acumulan 133 millones de euros de facturación. Con esta iniciativa se vislumbra una importante oportunidad de crecimiento, más aún con las últimas incorporaciones de las importantes cadenas Rewe (Alemania) y Ahold (Holanda), que debe permitir concretar acuerdos comerciales con las principales empresas proveedores multinacionales.

\section{CCFACC}

La Federación Argentina de Cooperativas de Consumo (FACC) reúne a las cooperativas de consumo argentinas, la cual llevó a cabo el proyecto de su central de compras iniciado el 6 de abril de 2016. La necesidad de la Central de Compras surgió en el 2014, cuando las cooperativas de consumo detectan el aumento de la competencia y, frente a las cadenas nacionales e internacionales, necesitan afrontar esa competencia.

De esta forma, surge el proyecto de la Central de Compras de la Federación Argentina de Cooperativas de Consumo (CCFACC), con el objetivo de, por un lado, administrar un sistema de compras en común para las cooperativas y mutuales de consumo, o con sección consumo, con la finalidad de conseguir para este sector de la economía social mejores condiciones comerciales, fortaleciendo su competitividad, mejorando y ampliando sus servicios a los asociados; y, por otro lado, incrementar y agilizar las relaciones comerciales entre las cooperativas de trabajo y/o producción y las cooperativas y mutuales de consumo o con sección consumo (según documentación interna de la CCFACC).

La operatoria de la CCFACC es de modo virtual a través de un portal de internet exclusivo para las cooperativas y mutuales adheridas a la central en donde se ofrecen los productos que forman parte del surtido ofrecido con toda la información relevante para su compra. Todas las condiciones (precios, forma de pago, lugares de entrega, etc.) son pactadas por la central y se ofrecen por igual a todas las cooperativas y/o mutuales participantes.

Tras un año de la puesta en marcha de la CCFACC, hay 38 entidades adheridas, de las cuales 24 se encuentran ya operando con dicha herramienta. Estas 
organizaciones representan 198 puntos de venta y más de $130.000 \mathrm{~m}^{2}$ de superficie de venta ubicados en 121 localidades a lo largo de todo el territorio argentino.

Un punto para destacar es que el número de cooperativas de consumo adheridas antes de la puesta en marcha era 17, lo cual significa que, en estos doce meses de funcionamiento, se han ido sumando otras 21.

Por el otro lado, están homologados 60 proveedores, dentro de los cuales, 32 son cooperativas de producción y trabajo y, de la mismas, 19 son proveedoras de las marcas Cooperativa, Ecoop y Coop. Más de mil referencias de productos se están trabajando de distintas categorías de almacén, bebidas, limpieza, perfumería, bazar y hogar.

\subsection{Fuentes de información}

En cuanto a la recolección de datos, se ha realizado un análisis tanto cuantitativo como cualitativo de la documentación interna de ambas entidades; como boletines, registros e informes de gestión de ventas y asociados, presentaciones, dossiers, entre otros.

A su vez, en Septiembre de 2016 se llevó a cabo en Bahía Blanca (Argentina) el Encuentro Intercooperativo organizado por la propia FACC, donde tanto las cooperativas de consumo como de producción y trabajo pudieron comentar sus experiencias y realizar comentarios sobre el funcionamiento de la central de compras.

Adicionalmente, también se han realizado entrevistas a representantes de la CCFACC (Presidente, Secretario y Consejero) y de la Coop Italia (Vice Presidente Vicario ANCC-COOP), cuando se visitaron las entidades. A su vez, se tomó como fuente de información secundaria, la entrevista a Barberini (2009), cooperativista italiano de referencia a nivel mundial, ex presidente de la Coop Italia y de la ACI.

\subsection{Análisis de datos}

Tomando como referencia a Cepeda Garrión (2006) en cuanto a que "el desafío práctico consiste en determinar cuáles van a ser los resultados reales de la investigación", aquí se ha considerado como objetivo comparar la información de las dos unidades de análisis con los problemas hallados en el marco teórico y ver si se corresponden la realidad de las centrales de compras cooperativas con la realidad de las redes de compras lucrativas.

Al objeto de poder obtener una síntesis de problemas y beneficios que reporta la central para las cooperativas participantes, se ha trabajado con el material recolectado durante los foros, con la información interna y la obtenida de las entrevistas a representantes de las centrales, realizando un análisis cualitativo y tratando de sintetizar la misma de manera objetiva.

En cuanto al análisis cualitativo de los datos, el mismo se ha realizado siguiendo los pasos detallados por Ryan y Bernard (2003): obtención de la información; capturación, transcripción y ordenamiento; codificación (agrupación por categorías) y, por último, integración de la información. 


\section{Resultados}

Habiendo recolectado la información de las dos centrales de compra (unidades de análisis), es posible identificar aquellos inconvenientes detectados para la buena investigación en cada caso en particular.

En primer lugar, en base al ciclo de vida de las centrales de compra establecido por Pousa (2006), se puede distinguir que la CCFACC se encuentra en una incipiente etapa de maduración, en donde la gestión ya está a cargo de personal contratado, se realizan las compras en conjunto y se comienza a contar con una oferta más amplia de servicios de marketing y asesoramiento, tratando de mejorar los procesos logísticos, sin llegar a tener abastecimiento centralizado.

Otra distinta es la realidad de la Coop Italia, ubicándose fuertemente en la madurez plena, con una gestión altamente profesional con sólidos conocimientos y un claro planeamiento estratégico, sobrepasando las fronteras nacionales, al recurrir a la importación y exportación de productos desde hace ya unos años. A su vez, cuenta con sistemas de información, logística y pagos totalmente integrados.

Esta distinción resulta de importancia, debido a que se trata del análisis de dos fases del ciclo de vida de las centrales, por lo cual los problemas presentados varían $\mathrm{y}$, en algunos casos, se comprueba que la Coop Italia atravesó por situaciones similares a las que se le están presentando a la CCFACC y, gracias al conocimiento y la experiencia, se encuentre la mejor manera de superarlas.

Cuadro. 3. Problemas teóricos hallados en cada una de las centrales

\begin{tabular}{|l|c|c|c|}
\hline Problema & CCFACC & Coop Italia & $\begin{array}{c}\text { No se } \\
\text { verificó }\end{array}$ \\
\hline Constitución societaria & & & $\mathrm{X}$ \\
\hline $\begin{array}{l}\text { Dificultad de establecer } \\
\text { mecanismos de control e } \\
\text { incentivos }\end{array}$ & & & $\mathrm{X}$ \\
\hline Doble tributación & $\mathrm{X}$ & & $\mathrm{X}$ \\
\hline $\begin{array}{l}\text { Asimetría de información } \\
\text { Diferencia de tamaño de los } \\
\text { participantes / Volumen de } \\
\text { pedidos }\end{array}$ & $\mathrm{X}$ & $\mathrm{X}$ (Actual) & \\
\hline $\begin{array}{l}\text { Proceso de gestión / Lentitud en } \\
\text { procesos decisorios }\end{array}$ & $\mathrm{X}$ & $\mathrm{X}$ (primera \\
\hline etapte-beneficio
\end{tabular}




\begin{tabular}{|l|c|c|c|}
\hline regionales & & & \\
\hline Logística & $\mathrm{X}$ & $\begin{array}{c}\mathrm{X} \text { (primera } \\
\text { etapa) }\end{array}$ & \\
\hline $\begin{array}{l}\text { Falta de apoyo del programa } \\
\text { público }\end{array}$ & & & $\mathrm{X}$ \\
\hline
\end{tabular}

Fuente: elaboración propia

En primer lugar, es posible identificar que en ninguno de los dos casos se explicita haber tenido problemas con la falta de apoyo del programa público, la doble tributación o la constitución societaria, cuestiones ligadas al marco jurídico de cada país. Así como tampoco surgen inconvenientes por expectativas de precios bajos, dificultad de establecer mecanismos de control o análisis coste-beneficio, aunque este último puede estar relacionado indirectamente con la falta de compromiso.

Por otro lado, es interesante observar la distinción de los problemas de la Coop en relación la etapa a la que pertenecen, para poder lograr una comparación más enriquecedora.

La falta de compromiso parece ser el inconveniente más recurrente entre las centrales de compra, por lo menos en una primera etapa, dado que en ambos casos estudiados se destaca y en la bibliografía también. Esta ausencia de compromiso puede entenderse como consecuencia de una falta de confianza en el modelo, lo cual es lógico que se dé en las etapas iniciales, cuando todavía no hay suficientes resultados comprobados.

Según testimonios de la CCFACC, un factor que influye en este aspecto es la diferencia de tamaño, dado que se observa que las cooperativas más pequeñas están más centradas en resolver sus problemas actuales, y su mirada cortoplacista les impide visualizar los beneficios a futuro que se puede tener apostando a este modelo. Algo similar ocurrió en Italia en la crisis de 1973/5, cuando se detectó falta de compromiso por parte de las cooperativas debido a la objetiva debilidad de la Asociación Nacional y de la Coop, la cual supo manejar muy bien la crisis. Como afirma Barberini (2009), "Se dio el recambio de los grupos dirigentes a nivel nacional. La situación de crisis implicaba cambios radicales, que tenían que ver con todos y cada uno de los aspectos de la empresa" (p. 208). De esta forma, lograron crear un clima más positivo diseñado por personalidades con objetivos compartidos.

Relacionado con este tema, se encuentra el conflicto de intereses. Por ejemplo, en la CCFACC se visualiza este inconveniente desde el hecho de la no participación de algunas cooperativas o la participación parcial de otras cooperativas importantes que serían clave en el funcionamiento de la central. Según el consejero de la FACC, "hoy, uno de los desafíos más grandes que tenemos es que se asocien las cooperativas proveedoras grandes que nos van a marcar la diferencia, en surtido especialmente".

En este sentido, hay consenso en los dos casos en relación a que el convencimiento en el modelo es la clave para el éxito del mismo. Es necesario que todos crean y aporten para la misma causa, y no utilicen la central sólo para algún beneficio puntual y actúen de forma individualista. El presidente de la FACC hace 
hincapié en la importancia de la convicción, y que la fidelidad termina siendo una consecuencia de esta primera.

En los orígenes de la Coop Italia, el conflicto de intereses también salió a la luz en la crisis de 1973/5, momento de gran división en el grupo dirigente (Barberini, 2009). Un factor de discusión era, por ejemplo, las comisiones que el Consorcio Nacional cobraba. El riesgo de quiebra de muchas cooperativas fue muy alto, lo que implicó un proceso enorme de redimensionamiento con la pérdida de todos los avances que se habían logrado hasta el momento. Por otra parte, una particularidad del caso italiano es que el cooperativismo siempre estuvo muy ligado al Partido Comunista, lo cual implicó, en su momento, una adhesión partidaria consecuente; realidad que no sucede en Argentina ni en Italia en la actualidad.

Por otro lado, la diferencia de tamaño también se relaciona con el problema del volumen de los pedidos, dado que las cooperativas de menor tamaño encuentran dificultades para llegar a los mínimos logísticos necesarios. Según el Vicepresidente Vicario de la Coop, en la actualidad, la Coop está transitando un proceso de asociación de las cooperativas más pequeñas, ya que es la única salida posible que encuentran para poder subsistir. Según Barberini (2009), "la búsqueda de la optimización de las dimensiones llevó a reunir a las más de tres mil cooperativas en un número cada vez más reducidos de empresas más o menos grandes"(p. 185), afirmación que asegura que la diferencia de tamaño es un problema que está vigente desde los inicios.

Por el lado de la logística, debe tenerse presente que Argentina cuenta con una superficie de 2,78 millones de $\mathrm{km} 2$ e Italia de $300.000 \mathrm{~km} 2$, por lo que se están tratando dos realidades logísticas totalmente diferentes. Además de tener unas menores distancias, en Italia las vías de transporte son autopistas, mientras que en Argentina el diseño vial cuenta con rutas de un solo carril, en su mayoría. Además, se debe tener en cuenta que la CCFACC presenta un esquema logístico sin almacenes propios, cuando la organización de la Coop Italia es diferente. Al estar dividido por consorcios, cada consorcio tiene sus propios almacenes de distribución y la Coop entrega en estos almacenes, después, una cooperativa de transporte contratada se encarga de trasladar la mercadería a cada cooperativa (300 $\mathrm{km}$, aproximadamente, a la redonda como máximo).

Actualmente la logística no significa un problema para la Coop Italia, pero sí lo fue en sus inicios. Barberini (2009) asegura que se han realizado inversiones importantes para renovar la red logística y, en pocos años, se han concentrado los centros de aprovisionamiento y se han tecnificado.

Por otra parte, el surtido de productos es otro tema que se resalta en la CCFACC, la cual no cuenta con productos perecederos debido a las dificultades logísticas que conllevan. Una solución importante al problema de surtido observada en ambos casos es la marca propia. La Coop Italia tiene desarrollada la marca propia con más de 4.000 referencias, mientras que, en el caso de la CCFACC, actualmente se empiezan a comercializar los productos de las marcas propias de Cooperativa Obrera.

La Coop Italia, al encontrarse ya en una etapa de madurez plena, está en la fase de optimización de procesos, dedicada a encontrar la medida justa al negocio. A su vez, están muy pendientes de su competencia y no quieren perder cuota de mercado, por lo que son conscientes de la dificultad de reacción ante cambios en el 
mercado. Se observa que la competencia responde rápido, actúa y a la Coop ese cambio le cuesta más tiempo y, para cuando se lleva a cabo, muchas veces se ha perdido ya la oportunidad. Por la propia estructura de la Coop, cualquier proceso decisorio toma más tiempo que en la competencia en donde, en general, las decisiones están centralizadas, hecho que puede provocar pérdida de competitividad.

Desde el punto de vista de las cooperativas asociadas a la CCFACC, hay cuestiones a mejorar en cuanto a la información de los productos, de seguimiento de pedidos, de precios, entre otras; siendo aplicable esta realidad tanto a cooperativas de consumo como de producción y/o trabajo.

En lo que se refiere a organización y comunicación, la estructura de la Coop Italia ha ido mejorando con el transcurso del tiempo y hoy no es un problema. Existe una organización centralizada en relación a las decisiones y gestión de marca propia, imagen marca, comercio exterior, control de calidad, marketing y capacitación; de forma que todos los consorcios y cooperativas manejen la misma información. Aunque en la CCFACC se está planteado ese mismo modelo, todavía hace falta tiempo de funcionamiento para ajustar la comunicación y mejorar la imagen delante de los socios.

Por último, mencionar que en la Coop, en los años '80, se comenzaron a plantear temas sobre desarrollo estratégico del negocio, como la apertura o no de hipermercados o la unificación del nombre. Los mismos dieron lugar al debate y a posiciones bien marcadas. Una salida válida que encontró la Coop fue el asesoramiento de profesionales expertos, lo que dio lugar a una toma de decisiones según una lógica de sistema, no de una empresa individual. La CCFACC todavía no se ha encontrado con este problema, dado que recién comienzan a operar en conjunto.

\section{Conclusiones}

Siguiendo con lo propuesto por Pereira et. al. (2010), el presente trabajo ha aportado a la bibliografía el estudio de dos casos de centrales de compras cooperativas situadas en contextos diferentes y en distintas fases del ciclo de vida, distinguiendo los problemas con los que se enfrentaron y de qué forma lo solucionaron, con el objetivo de extraer buenas prácticas que faciliten la gestión de una red de compras cooperativa.

De acuerdo al análisis comparativo realizado, se comprueba que la mayoría de los problemas enunciados por la revisión bibliográfica se confirman en los casos de estudio. Por otro lado, resulta interesante concluir que en ninguna de las dos centrales se observan problemas de doble tributación, constitución societaria o falta de apoyo público, todos ellos relacionados con el marco jurídico-político, aunque se trate de dos contextos totalmente diferentes.

En cuanto a las coincidencias entre los dos modelos, se nota que la falta de compromiso y de confianza es uno de los problemas más recurrentes. Además, se detecta que es un inconveniente propio de una primer etapa, dado que es algo que le está afectando a la CCFACC y ya lo transcurrió la Coop en sus inicios, por lo 
cual resulta necesario darle tiempo al proyecto para que consiga la madurez necesaria.

De la mano de la convicción también se puede relacionar la cuestión de conflicto de intereses. Una vez que se está de acuerdo con el modelo y se actúa hacia el logro de un bien común, no habría lugar para el conflicto de intereses. Eso sí, es necesario que la gestión de la central siempre sea prolija, transparente y coherente con lo planteado, dado que la cooperación termina cuando alguna de las partes percibe tratamiento injusto o resultados incompatibles con su contribución (Pereira et. al., 2010)

Por otro lado, la dispersión geográfica y la diferencia de tamaño de los participantes son características de la CCFACC que hoy pueden ser inconvenientes a su gestión óptima. Sin embargo, se observa que éstas también tuvieron lugar los primeros años de funcionamiento de la Coop, y la misma fue capaz de superar tales condiciones problemáticas. Por un lado, se instalaron almacenes propios y se subcontrató el transporte mediante la contratación de una cooperativa de transporte, al mismo tiempo que se favoreció la asociación de las cooperativas más pequeñas para llegar a un volumen de pedidos razonable para poder operar competitivamente.

"Errores y correcciones formaron parte de una etapa transitoria difícil, que resguardaba no sólo a la cooperativa, sino a toda la sociedad civil." (Barberini, 2009: p. 194). Observando el presente exitoso de la Coop Italia, con un $19 \%$ del total del mercado italiano (facturación) y en constante crecimiento, no queda otra alternativa que convencerse que el cooperativismo integrado es capaz de ser eficiente en un mercado competitivo y que, aunque se le presenten situaciones no deseadas, es posible encontrarle una solución y proseguir su implantación exitosa.

Tras analizar estos dos casos, tomando la Coop Italia como caso experimentado y exitoso y la CCFACC como testigo incipiente y en pleno desarrollo, es posible asegurar que la CCFACC sigue el camino de la central europea. Queda pendiente estudiar en un futuro el camino escogido por la CCFACC para resolver sus problemas, realizando un seguimiento del ciclo de vida de la misma, así como también sería interesante seguir analizando la maduración de la Coop Italia y los nuevos retos que se le van presentando.

\section{Referencias bibliográficas}

Acosta, M. y Verbeke, G. (2009) La cooperación como estrategia de desarrollo en redes asociativas. Pecvnia, $\mathrm{N}^{\circ}$ 9, pp. 1-25.

Adobor, H. y R. Mcmullen (2014) Strategic purchasing and supplier partnerships - The role or a third party organization. Journal of Purchasing \& Supply Management, $\mathrm{N}^{\circ} 20$, pp. 263-272.

Alianza Cooperativa Internacional (2013) Plan para una década cooperativa. Disponible en: http://www.aciamericas.coop/.

ANCECO (2016) Presente y futuro de las CCS españolas. Revista Central, №4.

Asociación Nacional de Centrales de Compras y Servicios. Disponible en: http://anceco.wpengine.com/ Fecha de consulta: Enero de 2017.

Barberini, I. (2009) El Vuelo del Abejorro: Cooperativismo, Ética y Desarrollo. Argentina: Intercoop Editora Cooperativa Limitada. 
Cepeda Carrión, G. (2006) La calidad en los métodos de investigación cualitativa: principios de aplicación práctica para estudios de casos. Cuadernos de Economía y Dirección de la Empresa, $\mathrm{N}^{\mathrm{o}} 29$, pp. 57-82

Charterina, A. M. (2012) Sobre el principio de cooperación entre cooperativas en la actualidad. Boletín de la Asociación Internacional de Derecho Cooperativo, $\mathrm{N}^{\mathrm{o}} 46$, pp. 133-146.

Gummesson, E. (1991) Qualitative Research in Management. Qualitative Methods in Management Research. Londres: Sage Publications.

Heinz, D. et. al. (2014) Composto de marketing em redes de compras: estudo comparativo das percepcoes de administradores e associados emu ma rede de supermercados. Read, Porto Alegre, Edición 78, № 2, pp. 529-570.

Hernández Sampieri, R. et. al. (2010) Metodología de la investigación. Quinta edición. México: McGraw-Hill.

Masón, R. y Zoppi, R. (2013) El cooperativismo de consumo en la Argentina. Publicación $\mathrm{N}^{\circ} 17$, GIDECOOP UNS.

Menzani, T. y Zamagni, V. (2010) Cooperative networks in the Italian economy. Enterprise and Society, $\mathrm{N}^{\mathrm{o}} 11(1)$, p. 98.

Migliavacca, E. (2016) La Coop nell'attuale contesto competitivo. http://www.aciamericas.coop/ivcumbre.

Pereira, B. et. al. (2010) Desistencia da cooperacao e encerramento de redes interorganizacionais: em que momento essas abordagens se encontram? RAI: revista de administração e inovação, $\mathrm{N}^{\mathrm{o}} 7(1)$, pp. 62-83.

Pousa, R. (2006) Os Ciclos de Vida das Centrais de Negócios: Um Estudo no Setor Supermercadista no Estado de São Paulo. XIII SIMPEP-Simpósio de engenharia de produção. Bauru/SP, 6.

Ryan, G. y Bernard, H.R. (2003) Techniques to identify themes. Fied Methods, Vol. 15, No 1 , pp. 85-109.

Sena da Silva, E. (2016) Coop, a cooperativa da sua vida. http://www.aciamericas.coop/ivcumbre.

Souza, M. G. (2004) Centrais de Negócios. Uma revolução no varejo e na distribuição. São Paulo: Edições Inteligentes.

Xavier Filho, J. et. al. (2013) Desistencia da cooperacao em redes interorganizacionais horizontais: reflexoes a partir da tipología da acao social weberiana. XVI Seminarios em Administracao. Revista de Administração Mackenzie, $\mathrm{N}^{\circ} 16(6)$.

Yacuzzi, E. (2005) El estudio de caso como metodología de investigación: teoría, mecanismos causales, validación, Inomics, No 1, pp. 296-306.

Yin, R. K. (2001) Estudio de Caso. San Pablo: Artmed, 205.

Zsolnai, L. (2010) Future of capitalism. Ethics in the Economy - Handbook of Business Ethics, Oxford, Bern, Berlin: ed. Zsolnai, L., Lang, P. 